\title{
The Demonstration of Alternating Contractile State in Pulsus Alternans
}

\author{
R. Joe Noble and Donald O. NutTer with the assistance of \\ Harold J. CrumLy, JR. \\ From the Cardiovascular Research Laboratory, Department of Medicine, \\ Emory University School of Medicine, Atlanta, Georgia 30303
}

A B STRACT Pulsus alternans was induced in 11 anesthetized, open-chest dogs by rapid atrial pacing, and the left ventricular filling characteristics and lengthtension-velocity relationship of alternating beats were compared. The end-diastolic circumferences (circ) of the strong beats were slightly, but significantly, increased over the weak beats $(7.3>6.9 \mathrm{~cm}, P<0.01)$, confirming that diastolic filling does alternate in pulsus alternans. This alternation in initial fiber length seemed to result from an alternation in the prior end-systolic length, rather than from an alternation in diastolic filling time or compliance. There was also no difference in enddiastolic tension as measured by an isometric strain gauge suggesting no difference in contractile element relaxation before weak and strong beats.

The contractile state of the strong beats was consistently greater than that of the weak beats when contractility was defined in terms of: (a) $\mathrm{V}_{\max }(3.13>$ $2.53 \mathrm{circ} / \mathrm{sec}, P<0.01)$; and $(b)$ the velocity of circumferential fiber shortening $(0.84>0.39 \mathrm{circ} / \mathrm{sec}$, $P<0.001)$ and developed tension $(82.5>74 \mathrm{~g} / \mathrm{cm}$, $P<0.01)$ at isolength. The length-tension-velocity relationship of the left ventricle also varied between strong and weak beats when: (a) the maximum velocity of contractile element shortening at least common tension $(1.68>1.28 \mathrm{circ} / \mathrm{sec}, P<0.05)$; and $(b)$ the velocity of circumferential fiber shortening $(0.81>0.39 \mathrm{circ} /$ sec, $P<0.001)$ at maximum developed tension were examined. Analysis of the length-tension-velocity characteristics of sequential beats at the onset of alternans in three dogs suggests that an alternation in contractility initiates alternans, with secondary alternations in ventricular filling. Cross-clamping of the aorta in three

Dr. Noble was an NIH Postdoctoral Trainee (Training Grant HEO5653). Dr. Nutter is a Senior Investigator of the Georgia Heart Association.

Received for publication 2 September 1969 and in revised form 14 November 1969. other dogs essentially eliminated the alternating changes in end-diastolic length and pressure, while the resultant isovolumic contractions continued to demonstrate clear evidence of pulsus alternans in the ventricular systolic pressure, suggesting the persistance of an alternating contractile state. The evidence suggests that an important mechanism in the production and propagation of pulsus alternans, as produced in the intact canine ventricle by rapid pacing, is a beat-to-beat alternation in contractile state with secondary alternations in ventricular filling.

\section{INTRODUCTION}

Mechanical alternans in isolated myocardium is said to result from an alternation in muscle fiber stretch (1) or from an alternation in the inotropic state of the muscle $(2,3)$. Similarly, studies in pulsus alternans in the intact heart have shown this phenomenon to be both associated with (4-6) and independent of $(7,8)$ alternations in ventricular filling or end-diastolic tension. The relative contribution of variations in contractile state and in initial fiber length, and tension to the pathogenesis of pulsus alternans in the intact heart is unknown since alternating beats have not been analyzed in terms of the myocardial length-tension-velocity relationship.

In the present study, pulsus alternans was induced in open-chest, anesthetized dogs by rapid pacing, and the length-tension-velocity relations of the alternating beats were compared. In this preparation an important mechanism of pulsus alternans was found to be beat-tobeat alternation in the myocardial contractile state.

\section{METHODS}

11 mongrel dogs, weighing between 15 and $30 \mathrm{~kg}$, were anesthetized with a mixture of $1 \%$ halothane and $99 \%$ oxygen, administered by a Harvard respiratory pump through an endotrachial tube. Respiratory rate and tidal volume were 
adjusted to maintain arterial blood gases within reasonable physiological levels $\left(\mathrm{PO}_{2}>70 \mathrm{~mm} \mathrm{Hg} ; \mathrm{PCO}_{2} 29-45 \mathrm{~mm} \mathrm{Hg}\right.$; $\mathrm{pH}$ 7.35-7.48).

The heart was exposed through a left thoracotomy incision and suspended in a pericardial cradle. A micromanometer catheter (Model MSD8, Telco, Gentilly, France) was positioned in the left ventricular (LV) cavity by retrograde catheterization from the left common carotid artery. The right ventricle (RV) was catheterized with a micromanometer catheter (Model SF-1, Statham Instruments, Inc., Oxnard, Calif.) from the left external jugular vein. A bipolar pacing catheter was passed from the right femoral vein and positioned in the right atrium. Electromagnetic flow probes (EMP-400 series, Carolina Medical Electronics, Inc., King, N. C.) were placed on the ascending aorta and main pulmonary artery. A pair of ultrasonic transducers were surgically implanted on opposing, anteroposterior, endocardial surfaces of the LV at the level of the first diagonal branch of the anterior descending coronary artery. A Walton-Brodie isometric strain gauge was sutured to the anterior wall of the left ventricle in eight dogs.

High fidelity LV and RV pressures were recorded from the micromanometer catheters. A zero reference level was obtained by positioning strain gauge manometers (model $\mathrm{Db} 23$, Statham), connected to the catheter lumens, at the anteroposterior mid-chest level. The characteristics of the micromanometers are described elsewhere (9). The WaltonBrodie strain gauges were extended between 50 and $75 \%$ of their resting length and uncalibrated wall tension measurements were obtained continuously. Aortic and pulmonary artery blood flow were recorded using a twin channel square wave electromagnetic flow meter (model 322, Carolina Medical Electronics). The dynamic characteristics of this flow meter have been previously determined (10). Flow probe calibration was performed in vitro by timing the constant flow of whole blood (4-11 liters/min) of varying hematocrits through hemodialysis tubing. Continuous measurements of an internal, anteroposterior, LV diameter was performed by an ultrasonic transit time technique. The method of ventricular dimension measurement by sonomicrometry, the calibration technique, and the electronic characteristics of this device are described elsewhere (11). In our laboratory the transducers and the sonomicrometer were tested with a mechanical oscillator (Hydraulic Oscillator, Beta Corp., St. Louis, Mo.) and demonstrated a constant amplitude response without phase shift over a frequency range of $1-10 \mathrm{~Hz}$. The electronic first derivatives of $\mathrm{LV}$ pressure and of $\mathrm{LV}$ internal diameter were obtained by active differentiation. The differentiators (differential operational amplifier ${ }^{\circ} \mathrm{P} 2 \mathrm{~A}$, Philbrick/Nexus Research, Dedham, Mass.) have been tested in our laboratory using a sine wave input signal, and are linear to approximately $500 \mathrm{~Hz}$ with a sharp roll-off above this level. The phase shift of the derivative was $90^{\circ} \pm 1^{\circ}$. Derivatives were calibrated graphically by setting the mean of the derivative (as determined by planimetry) equal to the calculated mean rate of change of the differentiated signal (obtained as the ratio of peak amplitude to the time for its development). The resultant mean value and a known zero point allow calibration of the linear derivative curve.

The signals described above were recorded on two Electronics for Medicine, Inc. photographic recorders running simultaneously at a paper speed of $200 \mathrm{~mm} / \mathrm{sec}$ (Model DR-8, White Plains, N. Y.). A common electrocardiographic tracing was recorded simultaneously on both recorders for timing purposes.

Pulsus alternans was induced in seven dogs by rapid atrial pacing (120-190 beats/min). In four additional dogs, alternans did not result from atrial pacing alone but was produced by elevating aortic pressure $(30-50 \mathrm{~mm} \mathrm{Hg})$ with methoxamine $(0.1 \mathrm{mg} / \mathrm{kg})$ in concert with rapid atrial pacing. In three dogs the onset of pulsus alternans following the initiation of rapid atrial pacing was recorded and the initial beats analyzed for variations in filling and inotropic state.

In three additional dogs, the isolated ascending aorta was acutely cross-clamped in the presence of sustained pulsus alternans. The resultant sequence of isovolumic beats were analyzed for ventricular dimensional changes and the presence of mechanical alternans.

Calculations. LV stroke volume was determined by planimetry of the aortic flow pulse during systolic ejection. Enddiastolic circumferential length (EDL) and end-systolic circumferential length (ESL) were calculated in centimeters as the products of the measured end-diastolic diameter and end-systolic diameter and $\pi$. During isovolumic contraction, the measured ventricular diameter increased over the enddiastolic measurement in some dogs and decreased in others. Isovolumic diameter changes presumably occur due to geometric rearrangement of the chamber during isovolumic contraction. The variable directional changes in diameter, in turn, probably reflect the placement of the ultrasonic crystals nearer the midplane (isovolumic increase due to outward bulging as shown in Fig. 1), or nearer the apex (isovolumic decrease due to wall thickening and inward bulging as shown in Fig. 5) of the ventricle. Static enddiastolic compliance was calculated as EDL/EDP in centimeters per $\mathrm{mm} \mathrm{Hg}$, where EDP equals the end-diastolic pressure in $\mathrm{mm} \mathrm{Hg}$.

The velocity of shortening of the contractile element $\left(\mathrm{V}_{\mathrm{ce}}\right)$ was calculated in circumferences per second at $5 \mathrm{msec}$ intervals during the phase of isovolumic contraction as $\mathrm{V}_{\text {ce }}=(\mathrm{dp} / \mathrm{dt}) / \mathrm{KP}(12)$, where $\mathrm{dp} / \mathrm{dt}=$ the electronic first derivative of LV pressure in $\mathrm{mm} \mathrm{Hg}$ per second. $\mathrm{K}$ is the modulus of elasticity of the series elastic element which is equal to 28 in the dog heart (13), and $P$ is the instantaneous $\mathrm{LV}$ pressure in $\mathrm{mm} \mathrm{Hg}$. Tension ( $\mathrm{T}$ ) was calculated at the same $5 \mathrm{msec}$ intervals in grams per centimeter of circumference as $T=(P \cdot D) / 4$, where $P=L V$ pressure in $\mathrm{g} / \mathrm{cm}^{2}$, and $\mathrm{D}=\mathrm{LV}$ internal diameter in centimeters. $\mathrm{V}_{\text {ce }}$ was plotted against $T$ and the curves were extrapolated manually to zero $T$ to estimate $V_{\max }$; i.e., the maximum $\mathrm{V}_{\text {ce }}$ attainable. A comparison of $\mathrm{V}_{\max }$ for the alternating beats of pulsus alternans was used to assess the contractile state of the two beats (14). $V_{c e}$ was also used to assess the ventricular performance of weak and strong beats by comparing $\mathrm{V}_{\text {ce }}$ at the least tension common to both beats (15).

The velocity of circumferential shortening $\left(V_{c f}\right)$ during ejection was calculated in centimeters per second as $\mathrm{V}_{\text {ef }}=$ $\mathrm{dD} / \mathrm{dt} \times \pi$, where $\mathrm{dD} / \mathrm{dt}$ is the electronic first derivative of LV diameter or in some dogs was the slope of a line tangent to $\mathrm{D}$ during rapid ejection. $\mathrm{V}_{\mathrm{ct}}$ was divided by the circumference in centimeters to normalize for variations in ventricular size, and was expressed as circumferences per second. Tension during ejection was calculated as during isovolumic contraction.

The contractile state of alternating beats was estimated by comparing $\mathrm{V}_{\mathrm{c}}$ and tension at equal lengths; i.e., at the isolength point on the dimension trace (16). All $\mathrm{D}, \mathrm{T}$, and $\mathrm{V}_{\mathrm{cr}}$ comparisons were performed during an interval from 10 misec before peak aortic flow to $30 \mathrm{msec}$ after peak aortic flow to assure that the length-tension-velocity relationship was determined during the maximum active state of the muscle (12). Ventricular performance was estimated by 
TABLE I

Pressure and Flow Alternations in Pulsus Alternans

\begin{tabular}{|c|c|c|c|c|c|c|c|c|c|}
\hline \multirow[b]{2}{*}{ Dog } & \multirow[b]{2}{*}{ Heart rate } & \multicolumn{2}{|c|}{ LV pressure } & \multicolumn{2}{|c|}{$\operatorname{Max} \mathrm{dp} / \mathrm{dt}$} & \multicolumn{2}{|c|}{ Stroke volume } & \multicolumn{2}{|c|}{ Peak flow } \\
\hline & & Strong & Weak & Strong & Weak & Strong & Weak & Strong & Weak \\
\hline & beats $/ \mathrm{min}$ & \multicolumn{2}{|c|}{$m m \mathrm{Hg}$} & \multicolumn{2}{|c|}{$\mathrm{mm} \mathrm{Hg} / \mathrm{sec}$} & \multicolumn{2}{|c|}{$m l$} & \multicolumn{2}{|c|}{$\mathrm{ml} / \mathrm{sec}$} \\
\hline 1 & 120 & 91 & 88 & 1152 & 1201 & 10.8 & 6.6 & 106 & 83 \\
\hline 2 & 174 & 111 & 101 & 1728 & 1505 & 17.7 & 6.8 & 200 & 116 \\
\hline 3 & 190 & 81 & 76 & 1084 & 917 & 17.3 & 4.3 & 187 & 50 \\
\hline 4 & 158 & 146 & 142 & 2146 & 1990 & 13.0 & 9.8 & 150 & 116 \\
\hline 5 & 190 & 78 & 72 & 1352 & 1242 & 11.3 & 6.0 & 133 & 93 \\
\hline 6 & 121 & 114 & 109 & 1259 & 1110 & 5.7 & 4.6 & 72 & 51 \\
\hline 7 & 190 & 113 & 100 & 2240 & 1970 & 8.5 & 0.0 & 105 & 0.0 \\
\hline 8 & 182 & 105 & 90 & 2165 & 1671 & 18.5 & 4.2 & 211 & 86 \\
\hline 9 & 183 & 141 & 131 & 2459 & 1852 & - & - & - & - \\
\hline 10 & 190 & 78 & 74 & 1141 & 995 & 9.4 & 6.4 & 113 & 84 \\
\hline 11 & 187 & 130 & 121 & 1690 & 1661 & 11.8 & 3.2 & 121 & 54 \\
\hline Mean & - & 107 & 100 & 1674 & 1465 & 12.4 & 5.2 & 140 & 74 \\
\hline $\pm 1 \mathrm{SD}$ & - & \pm 23 & \pm 22 & \pm 485 & \pm 373 & \pm 4.0 & \pm 2.5 & 44 & 34 \\
\hline$P$ value & - & \multicolumn{2}{|c|}{$<0.001$} & \multicolumn{2}{|c|}{$<0.01$} & \multicolumn{2}{|c|}{$<0.001$} & \multicolumn{2}{|c|}{$<0.001$} \\
\hline
\end{tabular}

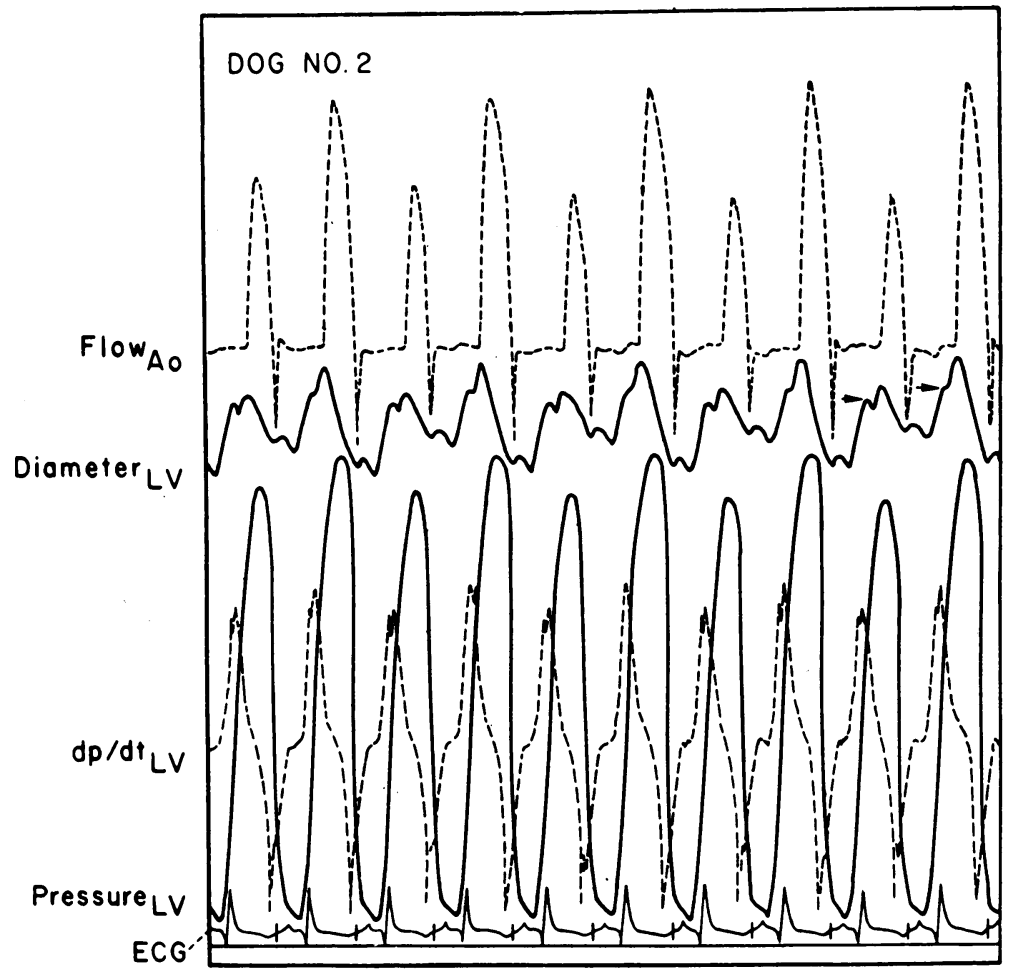

FIGURE 1 Left ventricular stroke volume, diameter, and pressure in pulsus alternans. All pressure and flow measurements are significantly greater for the strong beat. The end-diastolic diameter (illustrated by arrows) preceding the strong beat exceeds that preceding the weak beat. The slope of the diameter tracing during ejection reflects the rate of circumferential shortening. In this experiment the diameter increased during isovolumic contraction before decreasing with ejection. 
two methods: (a) $\mathrm{V}_{\text {ef, }} \mathrm{T}$, and $\mathrm{D}$ were compared at the respective maximum tensions of each beat; and $(b)$ in five dogs $\mathrm{V}_{\text {cf }}$ determined at $10 \mathrm{msec}$ intervals was plotted against $T$ calculated at $10 \mathrm{msec}$ intervals to produce a continuous force-velocity curve for ventricular ejection.

The present method of directly measuring a midplane ventricular dimension and its velocity of shortening obviates the geometrical assumptions required to mathematically derive dimension and velocity from a volume measurement. The calculation of tension per unit length of circumference at this ventricular level still rests on the relatively inaccurate assumption of a circular ventricular segment, and does not take into account resting wall thickness and its sequential change during ejection. The contractile state of only a segment of myocardium; i.e., the circumferential segment containing the ultrasonic crystals is evaluated by this technique.

The duration of isovolumic contraction (IVC) was defined and measured from the peak of the $R$ wave of the electrocardiogram to the beginning of forward aortic flow. The systolic ejection period (SEP) was measured on the aortic flow recording from the onset of forward flow to the point where the tracing returned to the preejection level. Isovolumic relaxation (IVR) was measured from the end of the SEP to the beginning of the diastolic filling period (DFP), as defined by a sharp increase in LV diameter. The DFP was measured from the end of IVR to the beginning of IVC. All measurements of time are expressed in milliseconds. The significant difference in the measured and calculated variables between the weak and strong beats of pulsus alternans was tested with a paired $t$ test (17).

\section{RESULTS}

Of the 11 dogs with alternans, 6 demonstrated concordant $\mathrm{LV}$ and $\mathrm{RV}$ alternans, 2 demonstrated discordan LV and RV alternans, and 3 showed LV alternans only. None of the dogs demonstrated an alternation in $\mathrm{RR}$ interval, $\mathrm{PR}$ interval, or in the electromechanical interval (as measured from the onset of the electrocardiographic $Q R S$ complex to the beginning of rapid $L V$ pressure rise).

The strong beat demonstrated, in comparison with the weak beat, a significantly greater peak LV pressure, $\mathrm{LV}$ maximal dp/dt, peak aortic flow, and stroke volume. These data are presented in Table I. Representative recordings of $\mathrm{LV}$ pressure, aortic blood flow, and $\mathrm{LV}$ internal diameter during pulsus alternans are illustrated in Fig. 1.

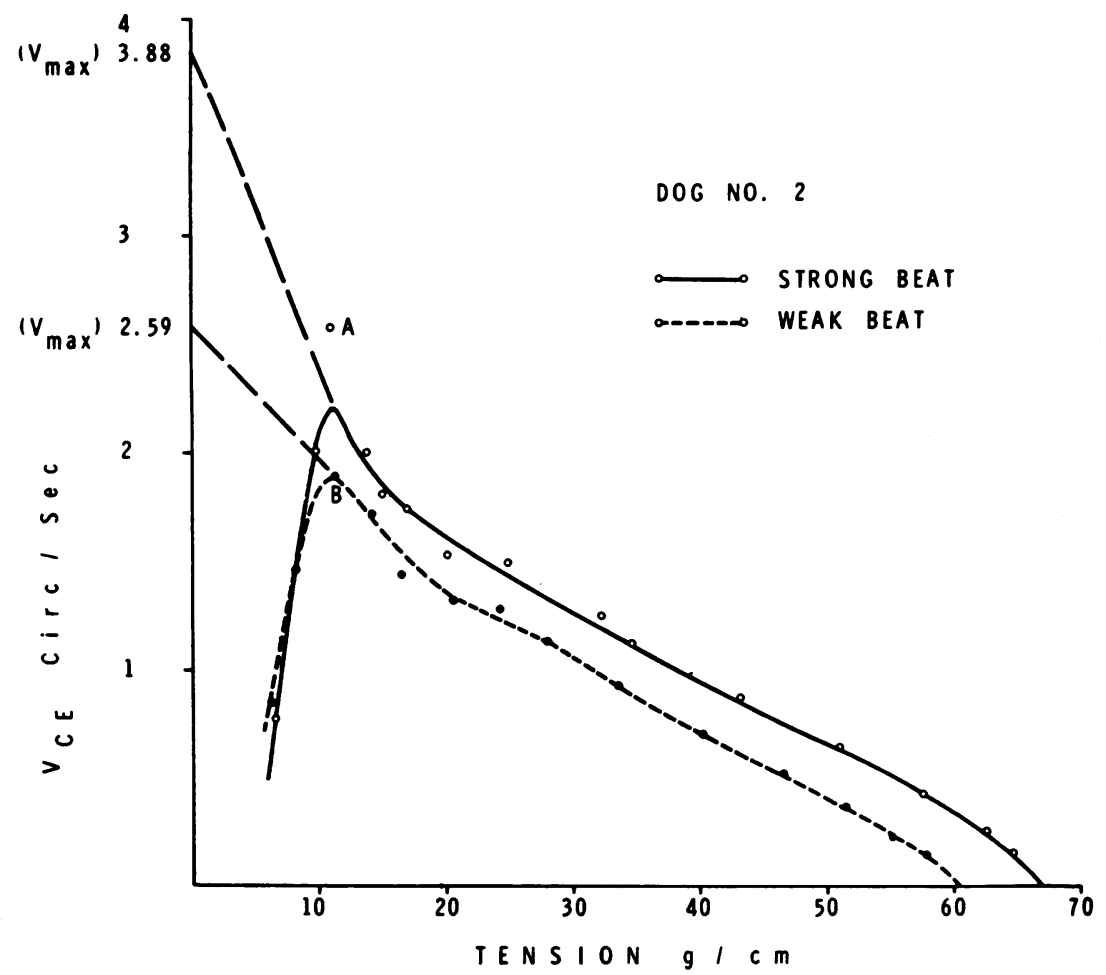

FIGURE 2 The relation of contractile element velocity $\left(V_{\mathbf{C E}}\right)$ to tension $(T)$ during isovolumic contraction in alternating beats. $\mathrm{V}_{\mathrm{CE}}$ increases at the onset of contraction until the contractile state is maximally active near the zenith of the curve, then diminishes reciprocally as $\mathrm{T}$ rises. $\mathrm{V}_{\mathbf{C r}}$ is manually extrapolated to zero $\mathrm{T}$ for each curve to compare $\mathrm{V}_{\max }$, which is demonstrated to be greater for the strong $(3.9 \mathrm{circ} / \mathrm{sec})$ than the weak beat $(2.6 \mathrm{circ} / \mathrm{sec})$. Points $A$ and $B$ indicate the maximum $\mathrm{V}_{\mathrm{cr}}$ of the strong $(2.6 \mathrm{circ} / \mathrm{sec})$ and weak $(1 / 9 \mathrm{circ} / \mathrm{sec})$ beats at the least $\mathrm{T}$ common to both beats. 
TABLE II

Comparison of Filling Char-

\begin{tabular}{|c|c|c|c|c|c|c|c|c|}
\hline \multirow[b]{2}{*}{ Dog } & \multicolumn{2}{|c|}{ IVC } & \multicolumn{2}{|c|}{ SEP } & \multicolumn{2}{|c|}{ IVR } & \multicolumn{2}{|c|}{ DFP } \\
\hline & Strong & Weak & Strong & Weak & Strong & Weak & Strong & Weak \\
\hline & \multicolumn{2}{|c|}{ msec } & \multicolumn{2}{|c|}{$m s e c$} & \multicolumn{2}{|c|}{ msec } & \multicolumn{2}{|c|}{$m s e c$} \\
\hline 1 & 80 & 85 & 185 & 175 & 112 & 102 & 132 & 125 \\
\hline 2 & 70 & 72 & 128 & 125 & 65 & 75 & 65 & 75 \\
\hline 3 & 58 & 68 & 145 & 132 & 78 & 78 & 68 & 70 \\
\hline 4 & 80 & 92 & 145 & 132 & 95 & 98 & 62 & 50 \\
\hline 5 & 62 & 72 & 142 & 112 & 78 & 82 & 42 & 50 \\
\hline 6 & 95 & 92 & 145 & 142 & 120 & 122 & 135 & 135 \\
\hline 7 & 72 & 85 & 140 & 108 & 70 & 80 & 35 & 32 \\
\hline 8 & 80 & 85 & 155 & 145 & 65 & 75 & 95 & 85 \\
\hline 9 & - & - & - & - & - & - & - & - \\
\hline 10 & 80 & 95 & 152 & 122 & 58 & 58 & 35 & 30 \\
\hline 11 & 72 & 98 & 148 & 95 & 82 & 100 & 48 & 38 \\
\hline Mean & 75 & 84 & 148 & 129 & 82 & 87 & 72 & 69 \\
\hline $\pm 1 \mathrm{sD}$ & \pm 10 & \pm 9.9 & \pm 14.1 & \pm 213 & \pm 19.8 & \pm 17.6 & \pm 35.4 & \pm 35.2 \\
\hline${ }^{*} P$ Value & \multicolumn{2}{|c|}{$<0.01$} & \multicolumn{2}{|c|}{$<0.01$} & \multicolumn{2}{|c|}{$<0.1$} & \multicolumn{2}{|c|}{$<0.1$} \\
\hline
\end{tabular}

* Significance of difference expressed between strong and weak beats.

IVC, isovolumic contraction period; SEP, systolic ejection period; IVR, isovolumic relaxation time; DFP, diastolic filling period; EDL, end-diastolic circumferential length; ESL, end-systolic circumferential length; EDP, enddiastolic pressure; IDP, instantaneous diastolic pressure of strong beat at diastolic isolength equal to the EDL of the weak beat.

In five dogs, stable nonalternating beats were recorded at heart rates which approximated those recorded during pulsus alternans. The mean stroke volume of the strong alternating beats $(13.8 \mathrm{cc})$ exceeded the mean stroke volume of the stable, control beats $(10.5 \mathrm{cc})$ at comparable heart rates. However, the weak alternating beat demonstrated such a significant reduction in stroke volume $(5.3 \mathrm{cc})$ that the cardiac output was reduced during pulsus alternans.

Ventricular filling relationships. Circumferential length at end-diastole (EDL) was consistently slightly greater before the strong (mean $7.3 \mathrm{~cm}$, range 4.6-11.7 $\mathrm{cm}$ ) than the weak beats (mean $6.9 \mathrm{~cm}$, range 4.4-10.7 $\mathrm{cm} P<0.01$ ). End-diastolic pressures (EDP) were quite variable, and there was no significant difference between the EDP initiating the strong (mean $15.6 \mathrm{~mm}$ $\mathrm{Hg}$; range 9.2-29.6 $\mathrm{mm} \mathrm{Hg}$ ) and weak beats (mean $15.5 \mathrm{~mm} \mathrm{Hg}$; range $8.2-28.1 \mathrm{~mm} \mathrm{Hg}$ ). However, there was not a significant alternation in end-diastolic compliance when defined either as ( $a$ ) EDL/EDP (strong beat $0.57 \mathrm{~cm} / \mathrm{mm} \mathrm{Hg}$, weak beat $0.53 \mathrm{~cm} / \mathrm{mm} \mathrm{Hg}$ ); or when $(b)$ diastolic pressures were compared at a length common to both beats, i.e., the EDL of the weak beat (instantaneous diastolic pressure of strong beats, mean $12.9 \mathrm{~mm} \mathrm{Hg}$, range $8.6-24.5 \mathrm{~mm} \mathrm{Hg}$, was not signifi- cantly different than that observed with weak beats, mean $15.1 \mathrm{~mm} \mathrm{Hg}$, range $8.1-28.1 \mathrm{~mm} \mathrm{Hg}$ ).

The strain gauge wall tension measurement at enddiastole did not differ before strong $(23.4 \mathrm{~mm})$ and weak $(23.7 \mathrm{~mm})$ beats. Since the myocardial segment under the strain gauge is presumptively maintained isometrically, it should not be affected by the small changes in ventricular dimensions that occurred before alternating beats in this study. A changing end-diastolic wall tension would, therefore, suggest a change in the degree of contractile element relaxation and the unvarying tensions in this study suggest no change in the extent of relaxation.

The end-systolic circumferential length was significantly less for the strong $(6.5 \mathrm{~cm}$, range $4.2-10.6)$ than the weak beats $(6.7 \mathrm{~cm}$, range $4.3-10.6, P<0.01)$. During ejection, circumferential length shortened an average of $11 \%$ with the strong beats, but only $2.9 \%$ with the weak beats.

The strong beats demonstrated a significantly shorter mean isovolumic contraction period $(75<84 \mathrm{msec}, P<$ $0.01)$ and a longer systolic ejection period $(148>129$ msec, $P<0.01$ ) than did the weak beats. There was no significant difference in the isovolumic relaxation time $(82<87 \mathrm{msec}, \mathrm{NS})$ since the rate of relaxation (nega- 
acteristics in Pulsus Alternans

\begin{tabular}{|c|c|c|c|c|c|c|c|c|c|}
\hline \multicolumn{2}{|c|}{ EDL } & \multicolumn{2}{|c|}{ ESL } & \multicolumn{2}{|c|}{ EDP } & \multicolumn{2}{|c|}{ EDL/EDP } & \multicolumn{2}{|c|}{ IDP } \\
\hline Strong & Weak & Strong & Weak & Strong & Weak & Strong & Weak & Strong & Weak \\
\hline \multicolumn{2}{|c|}{$\mathrm{cm}$} & \multicolumn{2}{|c|}{$\mathrm{cm}$} & \multicolumn{2}{|c|}{$m m \mathrm{Hg}$} & \multicolumn{2}{|c|}{$\mathrm{cm} / \mathrm{mm} \mathrm{Hg}$} & \multicolumn{2}{|c|}{$m m H g$} \\
\hline 6.91 & 6.62 & 5.89 & 5.99 & 29.6 & 28.1 & 0.23 & 0.235 & 24.5 & 28.1 \\
\hline 5.24 & 4.98 & 4.27 & 4.69 & 11.0 & 10.0 & 0.48 & 0.50 & 11.0 & 10.0 \\
\hline 11.69 & 10.70 & 10.61 & 10.61 & 17.3 & 20.5 & 0.68 & 0.52 & \multicolumn{2}{|c|}{$\begin{array}{l}\text { No isolength in } \\
\text { end-diastolic }\end{array}$} \\
\hline 6.36 & 6.33 & 5.67 & 5.75 & 24.4 & 25.5 & 0.26 & 0.25 & 22.4 & 25.5 \\
\hline 5.07 & 4.90 & 4.28 & 4.76 & 9.2 & 8.2 & 0.55 & 0.60 & 2.6 & 8.2 \\
\hline 8.24 & 8.05 & 7.40 & 7.52 & 11.4 & 10.9 & 0.76 & 0.74 & 8.9 & 10.9 \\
\hline 7.65 & 6.78 & 6.72 & 6.90 & 11.5 & 14.0 & 0.66 & 0.48 & \multicolumn{2}{|c|}{$\begin{array}{l}\text { No isolength in } \\
\text { end-diastolic }\end{array}$} \\
\hline 8.79 & 8.42 & 8.35 & 8.22 & 14.0 & 12.5 & 0.63 & 0.67 & 14.0 & 12.5 \\
\hline 6.96 & 6.28 & 5.74 & 6.18 & 9.5 & 10.0 & 0.73 & 0.63 & 12.0 & 10.0 \\
\hline 4.57 & 4.38 & 4.20 & 4.26 & 26.0 & 23.0 & 0.18 & 0.19 & 12.0 & 23.0 \\
\hline 8.85 & 8.59 & 8.33 & 8.45 & 7.6 & 8.1 & 1.16 & 1.06 & 8.6 & 8.1 \\
\hline 7.30 & 6.91 & 6.50 & 6.66 & 15.6 & 15.5 & 0.57 & 0.53 & 12.9 & 15.1 \\
\hline \pm 1.97 & 1.80 & \pm 1.94 & \pm 1.83 & \pm 7.3 & \pm 7.0 & \pm 0.27 & \pm 0.24 & \pm 6.4 & \pm 7.6 \\
\hline \multicolumn{2}{|c|}{$<0.01$} & \multicolumn{2}{|c|}{$<0.01$} & \multicolumn{2}{|c|}{$<1.0$} & \multicolumn{2}{|c|}{$<0.3$} & \multicolumn{2}{|c|}{$<0.2$} \\
\hline
\end{tabular}

tive $\mathrm{dp} / \mathrm{dt}$ ) was greater for the strong beat, but the extent of pressure drop was also greater. There was also no significant difference in the period available for diastolic filling $(72>69 \mathrm{msec}, \mathrm{NS})$ between the two beats. Data from individual dogs with the mean and one standard deviation for variables related to ventricular filling and the systolic time intervals are presented in Table II.

The force-velocity relation during isovolumic contraction. A plot of $\mathrm{V}_{\text {ce }}$ and $\mathrm{T}$ during isovolumic contraction in an individual dog with pulsus alternans is shown in Fig. 2. Vie was manually extrapolated to zero $\mathrm{T}$ to approximate $\mathrm{V}_{\max }$. In this example, and in all nine dogs in which adequate curves were available for extrapolation, $V_{\max }$ was greater for the strong beat. $V_{\max }$ averaged 3.13 circumferences per second ( $\mathrm{circ} / \mathrm{sec}$ ) and ranged from 1.61 to $5.36 \mathrm{circ} / \mathrm{sec}$ for the strong beats. These values were significantly greater $(P<0.01)$ than those for $\mathrm{V}_{\max }$ of the weak beats, which averaged 2.53 $\mathrm{circ} / \mathrm{sec}$, and ranged from 1.50 to $3.81 \mathrm{circ} / \mathrm{sec}$. The maximum $\mathrm{V}_{\text {co }}$ at the least $\mathrm{T}$ common to both beats was consistently and significantly $(P<0.05)$ greater for the strong (mean $1.68 \mathrm{circ} / \mathrm{sec}$; range $1.04-2.93 \mathrm{circ} / \mathrm{sec}$ ) than the weak beats (mean $1.28 \mathrm{circ} / \mathrm{sec}$; range 0.08 $1.89 \mathrm{circ} / \mathrm{sec}$ ). Individual data with the means and one standard deviation for $\mathrm{V}_{\max }$ and $\mathrm{V}_{\text {co }}$ at least common tension are presented in Table III.

The length-tension-velocity relation during ejection. Tracings of LV pressure, diameter, and rate of change of diameter of alternating beats in an individual dog are reproduced in Fig. 3, and are superimposed to indicate the relationships at systolic isolength. At isolength, i.e. when the diameter of the alternating beats is equal, the comparative tensions are functions of ventricular pressure. In Fig. 3 the pressure and therefore the tension, of the strong beat exceeds that of the weak beat. $V_{c r}$, whether determined by electronic differentiation or by constructing a tangent to the dimension tracing is also substantially greater for the strong beat. In all 10 dogs in which adequate isolength points were available, the $\mathrm{T}$ and $\mathrm{V}_{\mathrm{CF}}$ were greater for the strong beat than the weak beat by an average of 12 and $136 \%$, respectively. The differences in $T$ were small $(82.5>74.0 \mathrm{~g} / \mathrm{cm})$ but significant $(P<0.01)$. The values for $\mathrm{V}_{\mathrm{cr}}$ of the strong beat (mean $0.84 \mathrm{circ} / \mathrm{sec}$; range $0.50-1.39$ ) were significantly greater $(P<0.001)$ than those of the weak beats (mean $0.39 \mathrm{circ} / \mathrm{sec}$; range $0-0.74$ ).

When the length-tension-velocity relationship was assessed at the time of maximal developed tension, the length of the strong beat (mean $7.24 \mathrm{~cm}$; range 5.08- 
TABLE III

Comparison of Length-Tension-Velocity Relationships in Alternating Beats

\begin{tabular}{|c|c|c|c|c|c|c|c|c|c|c|c|c|c|c|}
\hline \multirow[b]{3}{*}{ Dog } & \multicolumn{8}{|c|}{ Systolic isolength determinations } & \multicolumn{6}{|c|}{ Maximum tension determination } \\
\hline & \multicolumn{2}{|c|}{$V_{\max }$} & \multicolumn{2}{|c|}{ Max $V_{c e}$} & \multicolumn{2}{|c|}{ Vcf } & \multicolumn{2}{|c|}{ Tension } & \multicolumn{2}{|c|}{ Vcf } & \multicolumn{2}{|c|}{ Tension } & \multicolumn{2}{|c|}{ Length } \\
\hline & Strong & Weak & Strong & Weak & Strong & Weak & Strong & Weak & Strong & Weak & Strong & Weak & Strong & Weak \\
\hline & \multicolumn{2}{|c|}{ circ/sec } & \multicolumn{2}{|c|}{$\mathrm{circ} / \mathrm{sec}$} & \multicolumn{2}{|c|}{$\mathrm{circ} / \mathrm{sec}$} & \multicolumn{2}{|c|}{$\mathrm{g} / \mathrm{cm}$} & \multicolumn{2}{|c|}{$\operatorname{circ} / \mathrm{sec}$} & \multicolumn{2}{|c|}{$\mathrm{g} / \mathrm{cm}$} & \multicolumn{2}{|c|}{$\mathrm{cm}$} \\
\hline 1 & - & - & - & - & 0.63 & 0.54 & 65 & 63 & 0.60 & 0.54 & 65 & 64 & 6.46 & 6.56 \\
\hline 2 & 3.88 & 2.59 & 2.57 & 1.89 & 1.11 & 0.59 & 58 & 53 & 1.11 & 0.65 & 58 & 54 & 5.08 & 5.30 \\
\hline 3 & 2.82 & 2.22 & 1.08 & 0.08 & - & - & - & - & 0.65 & 0.15 & 98 & 87 & 11.3 & 10.7 \\
\hline 4 & 2.72 & 2.32 & 1.36 & 1.25 & 0.67 & 0.34 & 96 & 94 & 0.67 & 0.34 & 96 & 94 & 6.31 & 6.31 \\
\hline 5 & - & - & - & - & 1.39 & 0.74 & 42 & 40 & 1.37 & 0.74 & 43 & 40 & 5.12 & 5.05 \\
\hline 6 & 1.61 & 1.50 & 1.04 & 0.94 & 0.63 & 0.45 & 122 & 95 & 0.63 & 0.45 & 129 & 95 & 7.64 & 7.69 \\
\hline 7 & 4.16 & 3.68 & 2.16 & 1.71 & 0.50 & 0.00 & 86 & 74 & 0.78 & 0.00 & 87 & 76 & 7.35 & 7.06 \\
\hline 8 & 5.36 & 3.81 & 2.93 & 1.75 & 0.89 & 0.18 & 95 & 85 & 0.89 & 0.18 & 95 & 85 & 8.73 & 8.73 \\
\hline 9 & 2.48 & 1.88 & 1.28 & 1.52 & 1.15 & 0.32 & 93 & 82 & - & - & - & - & - & - \\
\hline 10 & 2.18 & 1.94 & 1.09 & 0.86 & 0.76 & 0.54 & 47 & 44 & 0.72 & 0.54 & 50 & 46 & 6.00 & 5.77 \\
\hline 11 & 2.94 & 2.82 & 1.59 & 1.50 & 0.66 & 0.22 & 119 & 110 & 0.66 & 0.32 & 119 & 112 & 8.47 & 8.60 \\
\hline Mean & 3.13 & 2.53 & 1.68 & 1.28 & 0.84 & 0.39 & 82 & 74 & 0.81 & 0.39 & 84 & 75 & 7.24 & 7.17 \\
\hline $\pm 1 \mathrm{SD}$ & \pm 1.08 & \pm 0.75 & \pm 0.66 & \pm 0.54 & \pm 0.27 & \pm 0.22 & \pm 27 & \pm 22 & \pm 0.24 & \pm 0.22 & \pm 28 & \pm 22 & \pm 1.80 & \pm 1.68 \\
\hline$P$ Value & \multicolumn{2}{|c|}{$<0.01$} & \multicolumn{2}{|c|}{$<0.05$} & \multicolumn{2}{|c|}{$<0.001$} & \multicolumn{2}{|c|}{$<0.01$} & \multicolumn{2}{|c|}{$<0.001$} & \multicolumn{2}{|c|}{$<0.02$} & \multicolumn{2}{|c|}{$<0.4$} \\
\hline
\end{tabular}

$V_{m a x}$, velocity of contractile element shortening $\left(V_{c e}\right)$ extrapolated to zero tension; Max $V_{c e}$ at least tension common to both beats; $V_{0}$, velocity of circumferential shortening; Length, circumference length (diameter $\times \pi$ ).

11.3) was not significantly different from that of the weak beat (mean $7.17 \mathrm{~cm}$; range 5.05-10.7). The strong beats developed a maximum $\mathrm{T}$ (mean $84.1 \mathrm{~g} / \mathrm{cm}$; range 42.9-128.9) slightly but significantly greater $(P<0.02)$ than the weak beats (mean $75.5 \mathrm{~g} / \mathrm{cm}$; range 39.8-
112.5). The $\mathrm{V}_{\mathbf{c r}}$ was appreciably greater $(P<0.001)$ for the strong (mean $0.81 \mathrm{circ} / \mathrm{sec}$; range $0.6-1.37 \mathrm{circ} /$ $\mathrm{sec}$ ) than the weak beats (mean $0.39 \mathrm{circ} / \mathrm{sec}$; range $0-0.74 \mathrm{circ} / \mathrm{sec}$ ). On the average, the strong beats developed an $11 \%$ greater $\mathrm{T}$ and a $96 \%$ greater $\mathrm{V}_{\mathrm{CF}}$ than

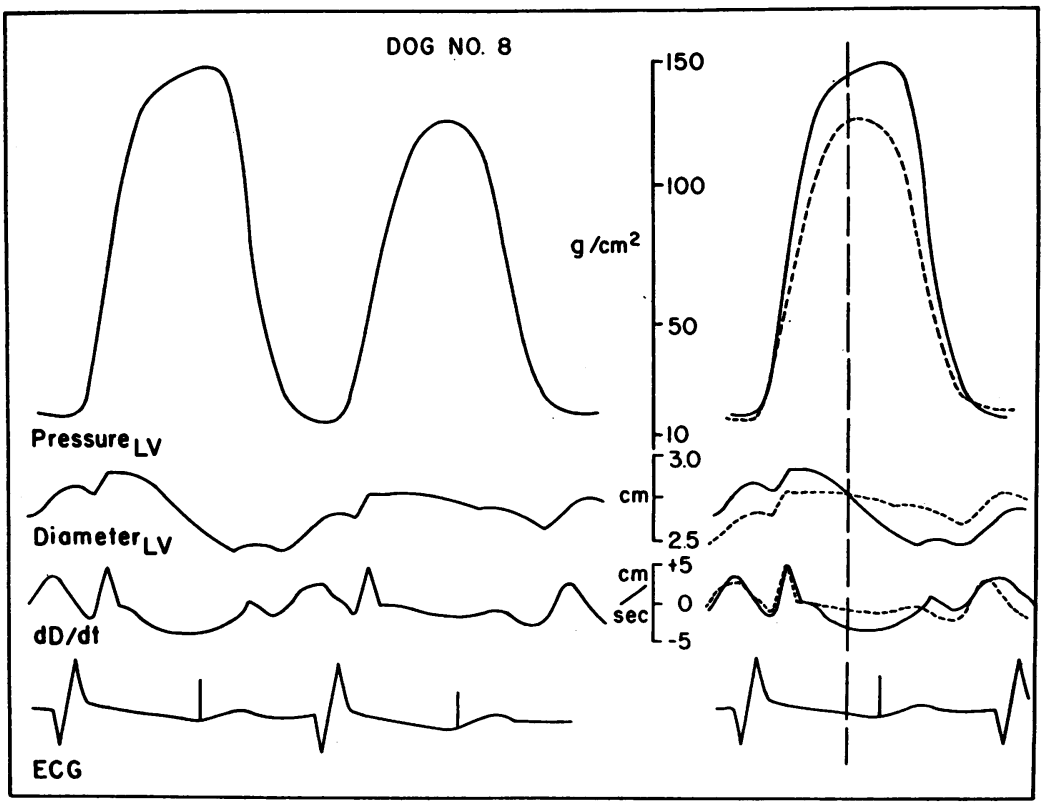

FIGURE 3 A comparison of the instantaneous force-velocity relations of the alternating beats at isolength. Reproduced tracings of the original signals on the left are superimposed on the right. The dashed lines indicate the systolic isolength point at which tension (a product of pressure and an equal dimension) and velocity (the product of $\mathrm{dD} / \mathrm{dt}$ and $\pi$ ) are compared between the two superimposed beats. Both the velocity of circumferential shortening and the tension are greater for the strong $(0.89 \mathrm{circ} / \mathrm{sec}$ and $95.1 \mathrm{~g} / \mathrm{cm})$ than the weak beat $(0.18 \mathrm{circ} / \mathrm{sec}$ and $85.0 \mathrm{~g} / \mathrm{cm})$ at isolength $(2.8 \mathrm{~cm})$. 


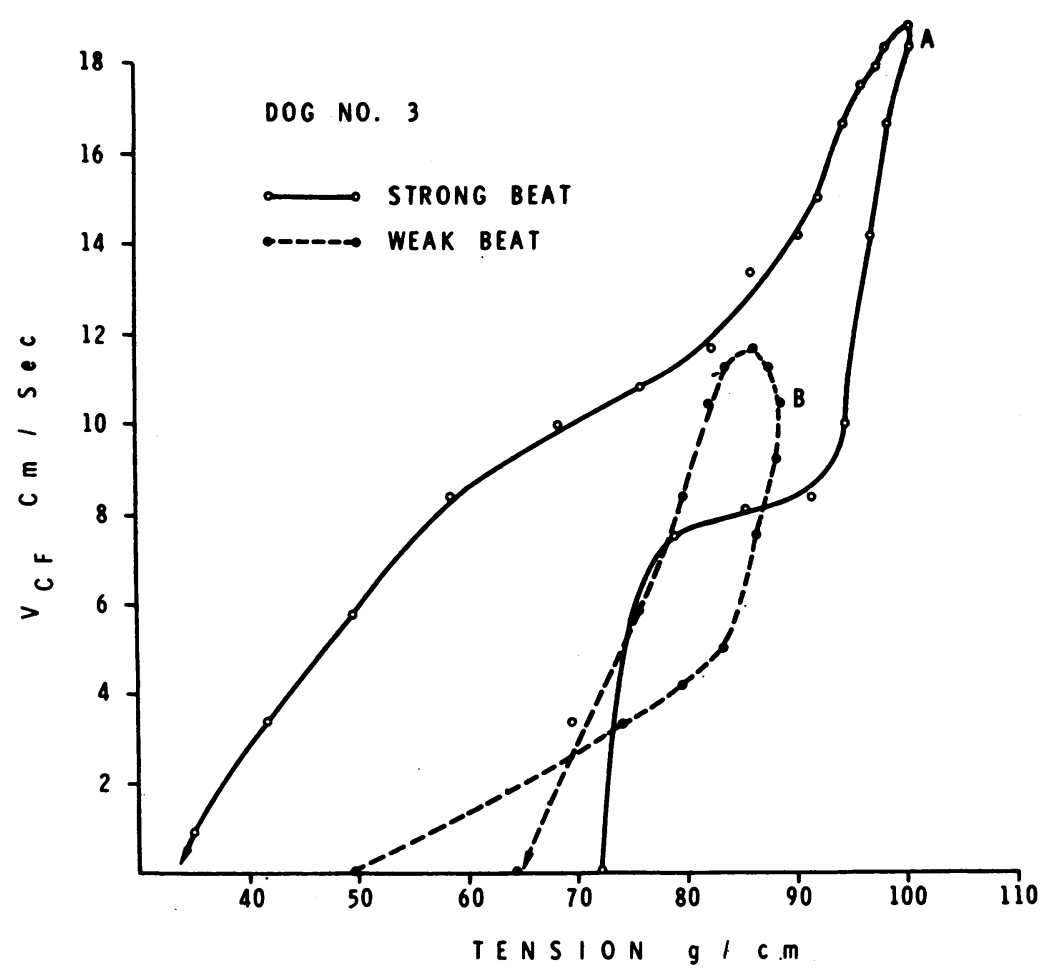

FIGURE 4 A comparison of the continuous force-velocity relations of the alternating beats during ejection. The velocity of circumferential shortening $\left(V_{C F}\right)$ is plotted against instantaneous tension $(T)$ for each of the beats of pulsus alternans in one representative animal. The curves are discussed in the text.

the weak beats. Individual data for the variables which characterize the length-tension-velocity relationship during ejection can also be found in Table III.

Continuous plots of the instantaneous force-velocity relation of the alternating beats in one representative dog with pulsus alternans are compared in Fig. 4. The strong beat developed a slightly greater maximum tension and a significantly greater maximum rate of shortening. Near the point of maximum tension, the velocity of shortening continued to increase with the strong beat, but showed little appreciable increase with the weak beat. The curves relating the tension and the velocity of circumferential shortening for the strong beats were broader and more likely to be inscribed in a counterclockwise direction than the curves for the weak beats.

The sequence of events during the onset of pulsus alternans. The characteristic sequence recorded in three dogs during the development of alternans is illustrated in Fig. 5. The first paced beat appeared as a premature atrial contraction with an abortive ventricular pressure pulse which failed to eject. The subsequent period of diastolic filling was shortened and the rate of filling was probably impaired by the elevated diastolic pressure. The subsequent, strong beat was initiated from an equivalent or reduced end-diastolic length, but developed a greater pressure at a faster rate $(\mathrm{dp} / \mathrm{dt})$ and ejected a larger stroke volume than the control beat. Both developed tension and the velocity of circumferential shortening were greater at systolic isolength, which suggests post-extrasystolic potentiation of contractile state without augmented filling. The third paced beat necessarily originated from a reduced end-diastolic length since it filled from a reduced end-systolic volume. This beat, however, also demonstrated a reduction in contractile state since both the developed tension and the velocity of circumferential fiber shortening were diminished at isolength. This sequence of events indicates that pulsus alternans induced by tachycardia may result from an alternation in contractile state with secondary alternations in ventricular filling, i.e., the observed varia tions in ventricular filling may result from a contractility-mediated alternation of stroke volume.

The development of isovolumic contractions during pulsus alternans by acute aortic cross-clamping in three dogs permitted equilibration of the LV end-diastolic diameters between strong and weak beats. Minor geometrical changes persisted during the isovolumic and ejection phases of systole, probably in response to the 


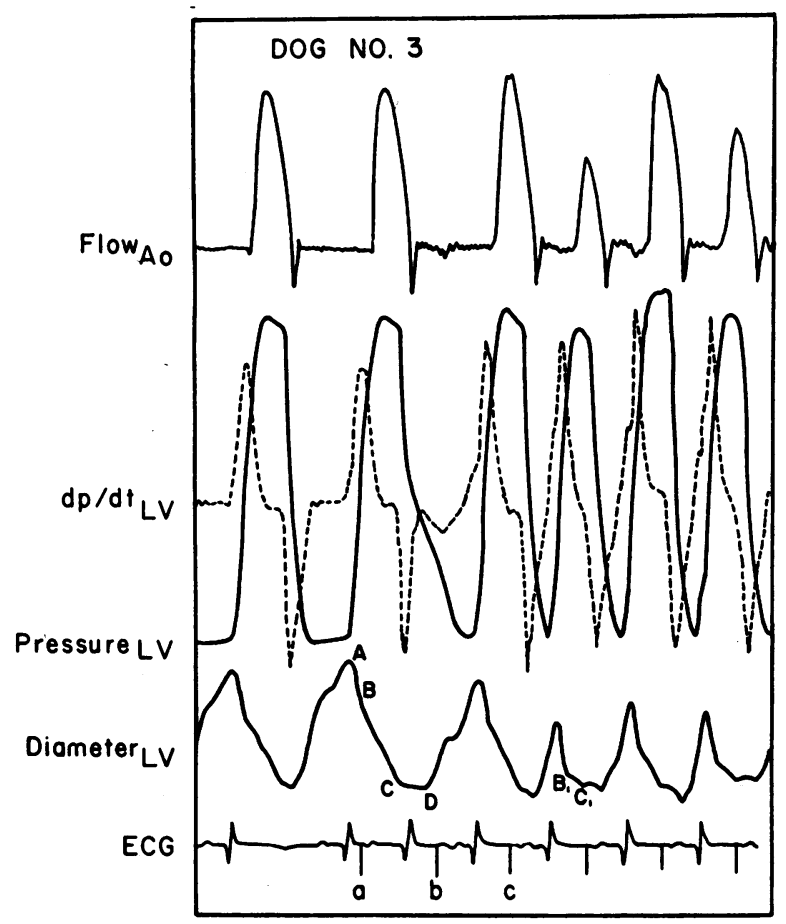

FIGURE 5 The length-tension characteristics of the initial beats at the onset of pulsus alternans. The first three consecutive atrial paced contractions which initiate pulsus alternans are labeled $a, b$, and $c$, respectively. Interval $A-B$ indicates isovolumic contraction and interval $B-C$ indicates ejection on the dimension tracing. The end-diastolic length (point $A$ on the dimension trace) of the second paced beat is decreased as performance is augmented by postextrasystolic potentiation (see text for details). In this experiment, in contrast to Fig. 1, left ventricular internal diameter decreases during isovolumic contraction.

presence of isovolumic chamber realignment and systolic coronary blood flow. The three dogs continued to demonstrate clear evidence of pulsus alternans in the ventricular pressure pulse despite the elimination of significant variation in chamber dimensions. Left ventricular dimension and pressure relationships before and after aortic cross-clamping are illustrated in Fig. 6.

\section{DISCUSSION}

Mechanisms based on both an alternation in contractile state and an alternation in end-diastolic length and tension have been proposed to explain the pathogenesis of pulsus alternans. Vigorous debate continues as to which of the two phenomena constitutes the primary mechanism. Lendrum, Feinberg, Boyd, and Katz observed beat-to-beat alternation in peak-developed tension in an intact heart preparation that was maintained in isovolumic state (7). Since their preparation permitted no variation in ventricular filling they concluded that the alternation in tension depended upon an alternation in the contractile state of the myocardium. Mitchell, Sarnoff, and Sonnenblick cast some doubt on this interpretation with their observation that an isolated papillary muscle strip maintaining itself isometric during mechanical alternans always developed a greater resting tension before the weak beat (1). Since muscle length remained constant, the greater resting tension before the weak beat was thought to result from a greater stretch of the series elastic element by a shorter contractile element. Thus, the weak beat originated from a reduced contractile element length and alternans in this circumstance could be explained by variation in the number of available contractile sites. The same authors also reported that in the intact heart end-diastolic volume was always less before the weak beat, regardless of end-diastolic pressure. End-diastolic wall tension in both the right and left ventricles of the intact dog heart was shown to be elevated before the weak beats in both concordant and discordant alternans (6). These authors also found an inverse relationship between ED wall tension and pressure during alternans in some dogs. Two studies of pulsus alternans in the intact human have employed cineangiography to demonstrate a reduced end-diastolic volume before the weak beat $(4,5)$. On the other hand, Cohn, Sandler, and Hancock found no variation in diastolic filling in two of three patients with pulsus alternans, thus suggesting that an alternation in a contractile state might occur in some patients during pulsus alternans (8). End-diastolic tension measurements were not available in the human studies listed above and changes in resting contractile element length cannot, therefore, be ruled out. The abolition of mechanical alternans in both isolated papillary muscle and in the intact heart with a variety of positive inotropic agents has suggested some indirect evidence for the existence of alternating inotropic state in the pathogenesis of mechanical alternans (18). Floyd and Dillon noted that pulsus alternans accompanied changes in rate and outflow resistance in the hypothermic dog heart where systolic relaxation was markedly prolonged and the diastolic filling period markedly shortened (19). Their data, however, does not distinguish between the presence of alternating end-diastolic tension or length and an alternating contractile state.

The detection and quantification of changes in myocardial contractile state and their relationship to altered ventricular filling can be best studied from the ventricular length-tension-velocity relationship (15). In the present study, the relationship was analyzed in an attempt to define the relative contribution of contractility and initial length in the pathogenesis of pulsus alternans associated with a rapid heart rate.

The present study does confirm a small, but con- 


\section{A. CONTROL \\ B. AORTIC OCCLUSION}

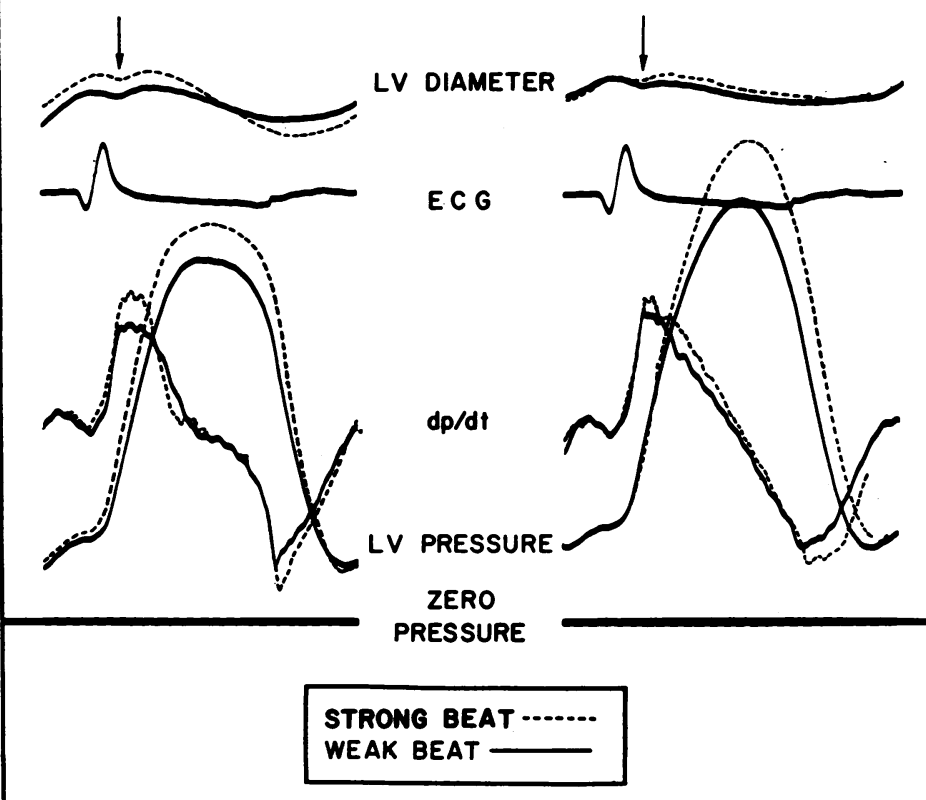

FIGURE 6 The effects of producing a series of isovolumic contractions during sustained pulsus alternans on left ventricular internal diameter, pressure, and $\mathrm{dp} / \mathrm{dt}$ are illustrated. Panel A contains superimposed alternating beats from one dog and shows the increase in end-diastolic length (arrow), peak LV pressure, and max dp/dt that occurs with the strong beat. (dotted line) Panel B contains superimposed beats recorded shortly after the ascending aorta was cross-clamped. The end-diastolic lengths (arrow) are equal, while pulsus alternans persists as indicated by the LV pressure events.

sistent alternation in ventricular filling in the intact heart with pulsus alternans, since the end-diastolic length initiating the strong beat was always slightly greater than the end-diastolic length of the weak beat. The observed differences in diastolic filling cannot be explained by a variation in time available for filling since the diastolic filling period of the strong beat did not significantly exceed that of the weak beat. A similar observation has been reported by Katz and Feil (20). The two indices of static end-diastolic compliance and the end-diastolic wall tensions calculated from our data do not reveal a significant difference between the alternating beats. The evidence, therefore, to support alternation in the degree of ventricular relaxation at enddiastole is not available. Dynamic compliance, however, was not evaluated in our preparation and previous studies have shown the difficulty in relying on a single static index of compliance to assess the impedance to ventricular filling or the sarcomere length $(21,22)$. Our data suggests, but does not prove, that the diminished enddiastolic length preceding the weak beats of pulsus al- ternans results from the significantly reduced end-systolic length of the previous strong beats.

The quantitative effect of a given variation in enddiastolic length on ventricular performance is difficult to predict. In the present study, the mean circumferential length of the strong beats at end-diastole $(7.3 \mathrm{~cm})$ was only $4 \mathrm{~mm}$, or approximately $5 \%$ greater than the mean end-diastolic circumferential length of the weak beats $(6.9 \mathrm{~cm})$. Extrapolating from two studies of the relation of muscle length to ventricular pressure and stroke volume in the human heart, it seems reasonable to assume that a $5 \%$ alternation in end-diastolic circumference would substantially influence ventricular performance $(4,23)$. In certain of our experiments, e.g. $\operatorname{dog} 4$, the minimal increase of $0.5 \%$ in end-diastolic length demonstrated by the strong beat hardly seems adequate to explain the $32 \%$ increase in stroke volume. An additional mechanism would appear to be operative in the production of alternans in such examples.

In this study an analysis of the length-tension-velocity relationship of the alternating beats, both during iso- 
volumic contraction and during ejection, has demonstrated an alternation of contractile state in pulsus alternans. $V_{\max }$, which has been shown to depend upon contractile state but not upon initial length or load, (14) was consistently increased for the strong beat. This demonstration of an augmentation in contractility for the strong beat was supported when the forcevelocity relation was examined during ventricular ejection. The force-velocity relation at any instant in time is a function of the instantaneous muscle length (24); therefore, the measurement of tension and velocity in alternating beats at a common length during ejection should provide an additional comparison of the contractile state of the two beats (16). The greater velocity of circumferential shortening $\left(\mathrm{V}_{\mathrm{Cr}}\right)$ and the greater wall tension developed by the strong beat at systolic isolength indicates an enhanced contractile state. Furthermore, the greater tension developed by the strong beat at isolength implies a greater stretch of the series elastic element with a decrease in the length of the contractile element as compared to the weak beat. Since a shorter contractile element would tend to reduce the velocity of shortening, the observed increase in velocity of shortening during the strong beat at systolic isolength emphasizes the augmentation in contractility.

The augmented ejection performance of the strong beats was demonstrated by the increase in $V_{c F}$ at peak tension. The more rapid $\mathrm{V}_{\mathrm{CF}}$ of the strong beat in the presence of a greater peak tension and comparable circumferential lengths also suggests that the contractile state is augmented. The curves depicting the forcevelocity relation during ejection (Fig. 4) further serve to illustrate the differences in cardiac function and ventricular contractility in the alternating beats of pulsus alternans. The strong beat develops a greater maximum tension and velocity of circumferential fiber shortening. Because of the more rapid rate of circumferential fiber shortening, the dimensions of the ventricle decrease more rapidly during the strong beat. Tension, a function of dimension, thus decreases more rapidly with the strong beat. Consequently, the tension of the strong beat at the conclusion of ejection is considerably less than that developed at the initiation of ejection, whereas there is little difference in initial and terminal tension for the weak beat. The tension-velocity curve of the strong beat resembles that described for the normal heart, whereas the curve for the weak beat is similar to that described for the failing ventricle (25).

The demonstration of an alternating contractile state during sustained pulsus alternans, the sequential changes in contractile state during the onset of pulsus alternans, and the demonstration of a persistent mechanical alternans in the presence of sustained isovolumic ventricular contractions all suggest that pulsus alternans induced in the intact canine heart by rapid atrial pacing manifests a significant alternation in contractile state similar to that observed during rate-induced alternans in isolated myocardium $(2,3)$.

The demonstration of alternating contractility, as defined by our measurements, does not distinguish between a true alternation of contractile state and an alternation in the strength of contraction that could result from alternating deletion of some portion of the myocardial fibers from the contraction process. Although $\mathrm{V}_{\max }$ is theoretically independent of the number of contractile sites, the deletion of fibers would probably alter the constant of elasticity employed in the calculation of $\mathrm{V}_{\max }$ (26). Similarly, the force-velocity relation during ejection would be expected to vary with the number of fibers actively involved in contraction. Previous investigation, however, provides some evidence against the fiber deletion theory of alternans by recordig intracellular action potentials from a variety of sites with every impulse during mechanical alternans $(27,28)$.

Mechanical alternans in the isolated, isovolumic myocardial preparation has in several studies been accompanied by alternation of the intracellular action potential $(3,27,29)$. Similar alternations in the action potential configuration have been correlated with changes in contractile state $(3,30,31)$, and therefore suggest that mechanical alternans in the isolated myocardium is due to an alternating contractile state.

The association of an alternation in action potential with an alternation in contractile state should not suggest that the changes in the cellular membrane responsible for the action potential variations are also responsible for the alternation in mechanical performance (32). Indeed, the observed action potential changes occur during repolarization, after the onset of the ventricular contraction. It has been proposed that a more central event in the contraction process influences both the membrane electrical activity and mechanical performance through some common pathway (3).

A unifying concept of the pathogenesis of pulsus alternans must interrelate the alternations which occur in contractile state, membrane repolarization, and ventricular performance. The available evidence, from the present and previous studies, suggests that a beat-to-beat alternation in the basic, cellular contractile process, induced by the time limitations imposed by rapid pacing, may be manifested as an alternation in membrane electrical activity and ventricular performance, with the latter inducing an alternation in ventricular filling. Speculations on the nature of the mechanism by which time and the events of one contraction influence the inotropic state of the subsequent beat are beyond the scope of this paper. An understanding of this phenomenon must await a full explanation of the intervalstrength relationship and the basic contractile process itself. 


\section{ADDENDUM}

A current report by Gunderoth, Morgan, McGough, and Scher analyzes the dynamics of tachycardia-induced pulsus alternans in anesthetized dogs. These authors also conclude that heterometric autoregulation is not the primary mechanism in pulsus alternans. They propose that an alternate deletion of contraction, coupled with potentiation of the contracting beats, in a certain proportion of myocardial cells constitutes the pathophysiologic mechanism of pulsus alternans (33).

\section{ACKNOWLEDGMENTS}

This study was supported in part by a research grant from the Georgia Heart Association and PHS Grant HE07782.

\section{REFERENCES}

1. Mitchell, J. H., S. J. Sarnoff, and E. H. Sonnenblick. 1963. The dynamics of pulsus alternans: alternating enddiastolic fiber length as a causative factor. J. Clin. Invest. 42: 55

2. Naylor, W. G., and P. G. C. Robertson. 1965. Mechanical alternans and the staircase phenomenon in dog papillary muscle. Amer. Heart J. 70: 494.

3. Greenspan, K., R. E. Edmands, and C. Fisch. 1967. The relation of contractile enhancement to action potential change in canine myocardium. Circ. Res. 20: 311.

4. Gleason, W. L., and E. Braunwald. 1962. Studies on Starling's Law of the Heart. VI. Relationships between left ventricular end-diastolic volume and stroke volume in man with observations on the mechanism of pulsus alternans. Circulation. 25: 841.

5. Harris, L. C., Q. X. Nghiem, M. H. Schreiber, and J. M. Wallace. 1966. Severe pulsus alternans associated with primary myocardial disease in children. Circulation. 34: 948

6. Gilmore, J. P., W. J. Powell, T. P. Graham, and R. L. Clancy. 1967. Discordant pulsus alternans in dog heart. Amer. J. Physiol. 212: 1515.

7. Lendrum, B., H. Feinberg, E. Boyd, and L. N. Katz. 1960. Rhythm effects on contractility of the beating isovolumic left ventricle. Amer. J. Physiol. 199: 1115.

8. Cohn, K. E., H. Sandler, and E. W. Hancock. 1967. Mechanism of pulsus alternans. Circulation. 36: 372.

9. Franke, E. K. 1966. II. Physiologic pressure transducers. In Methods in Medical Research. R. F. Rushmer, editor. Year Book Medical Publishers, Inc. Chicago. 11: 137.

10. Goodman, A. H. 1966. Electronic dynamic calibration of electromagnetic flow-meters. J. Appl. Physiol. 21: 933.

11. Stegall, H. F., M. B. Kardon, H. L. Stone, and V. S. Bishop. 1967. A portable, simple sonomicrometer. J. Appl. Physiol. 23: 289.

12. Ross, J., Jr., J. W. Covell, E. H. Sonnenblick, and E. Braunwald. 1966. Contractile state of the heart characterized by force-velocity relations in variably after loaded and isovolumic beats. Circ. Res. 18: 149.

13. Sonnenblick, E. H. 1964. Series elastic and contractile elements in heart muscle: changes in muscle length. Amer. J. Physiol. 207: 1330.

14. Levine, H. J., and N. A. Britman. 1964. Force-velocity relations in the intact dog heart. J. Clin. Invest. 43: 1383.

15. Covell, J. W., J. Ross, Jr., E. H. Sonnenblick, and E. Braunwald. 1966. Comparison of the force-velocity rela- tion and the ventricular function curve and measures of the contractile state of the intact heart. Circ. Res. 19: 364.

16. Glick, G., E. H. Sonnenblick, and E. Braunwald. 1965. Myocardial force-velocity relations studied in intact unanesthatized man. J. Clin. Invest. 44: 978.

17. Snedecor, G. W., and W. G. Cochran. Statistical Methods. Iowa University Press, Ames. 6th edition. 91.

18. Badeer, H. S., U. Y. Ryo, W. F. Gassner, E. J. Kass, J. Cavaluzzi, J. L. Gilbert, and C. McC. Brooks. 1967. Factors affecting pulsus alternans in the rapidly driven heart and papillary muscle. Amer. J. Physiol. 213: 1095.

19. Floyd, W. L., and M. L. Dillon. 1967. Observations on sustained pulsus alternans during hypothermia. Amer. Heart J. 73: 765 .

20. Katz, L. N., and H. S. Feil. 1937. Clinical observations on the dynamics of ventricular systole. IV. Pulsus alternans. Amer. J. Med. Sci. 194: 601.

21. Hoffman, B. F., A. L. Bassett, and H. J. Bartelstone. 1968. Some mechanical properties of isolated mammalian cardiac muscle. Circ. Res. 23: 291.

22. Noble, M. I. M., E. N. C. Milne, R. J. Goerke, E. Carsson, R. J. Domenech, K. B. Saunders, and J. I. E. Hoffman. 1969. Left ventricular filling and diastolic pressurevolume relations in the conscious dog. Circ. Res. 24: 269.

23. Braunwald, E., R. L. Frye, M. M. Aygen, and J. W. Gilbert. 1960. Studies on Starling's Law of the Heart. III. Observations in patients with mitral stenosis and atrial fibrillation on the relationship between left ventricular end-diastolic segment length, filling pressure, and the characteristics of ventricular contraction. $J$. Clin. Invest. 39: 1874.

24. Sonnenblick, E. H. 1965. Instantaneous force-velocitylength determinants in the contraction of heart muscle. Circ. Res. 16: 441.

25. Gault, J. H., J. Ross, Jr., and E. Braunwald. 1968. The contractile state of the left ventricle in man: instantaneous tension-velocity-length relations in patients with and without disease of the left ventricular myocardium. Circ. Res. 22: 451.

26. Forwand, S. A., K. M. McIntyre, J. G. Lipana, and H. J. Levine. 1966. Active stiffness of the intact canine left ventricle. Circ. Res. 19: 970.

27. Bravény, P. 1964. The relation of alternating contractility of the heart to the inotropic effects of rhythm. Arch. Int. Phy'siol. Biochem. 72: 553

28. Hogencamp, C. E., M. Kardesch, W. H. Danforth, and R. J. Bing. 1959. Transmembrane electrical potentials in ventricular tachycardia and fibrillation. Amer. Heart J. $57: 214$.

29. Kleinfeld, M., E. Stein, and J. Magin. 1956. Electrical alternans in single ventricular fibers of the frog heart. Amer. J. Physiol. 187: 139.

30. Muller, P. 1965. Ouabain effects on cardiac contraction, action potential, and cellular potassium. Circ. Res. 17: 46.

31. Prasad, K., and J. C. Callaghan. 1969. Effects of replacement of potassium by rubidium on the transmembrane action potential and contractility of human papillary muscle. Circ. Res. $24: 157$.

32. Sleator, W., Jr., R. F. Furchgott, T. De Gibareff, and V. Krepsi. 1964. Action potentials of guinea pig atria under conditions which alter contraction. Amer. J. Physiol. 206: 270.

33. Gunderoth, W. G., B. C. Morgan, G. A. McGough, and A. M. Scher. 1969. Alternate deletion and potentiation as the cause of pulsus alternans. Amer. Heart J. 78: 669 . 